\title{
BMJ Open Violence-related deaths among people released from incarceration: protocol for a systematic review
}

\author{
Melissa Willoughby (D) ,1,2 Jesse T Young (D) , ${ }^{1,2,3,4}$ Matthew J Spittal, ${ }^{1}$ \\ Rohan Borschmann, ${ }^{1,2,5,6}$ Emilia K Janca, ${ }^{1}$ Stuart A Kinner ${ }^{1,2,7,8,9}$
}

To cite: Willoughby M, Young JT, Spittal MJ, et al. Violence-related deaths among people released from incarceration: protocol for a systematic review. BMJ Open 2021;11:e045601. doi:10.1136/ bmjopen-2020-045601

- Prepublication history for this paper is available online. To view these files, please visit the journal online (http://dx.doi org/10.1136/bmjopen-2020045601).

Received 06 0ctober 2020 Revised 11 December 2020 Accepted 16 December 2020

A) Check for updates

(c) Author(s) (or their employer(s)) 2021. Re-use permitted under CC BY-NC. No commercial re-use. See rights and permissions. Published by BMJ.

For numbered affiliations see end of article.

\section{Correspondence to} Ms Melissa Willoughby; mwilloughby@student.unimelb. edu.au

\section{ABSTRACT}

Introduction Young people and adults released from incarceration have a risk of dying from violence that far exceeds that in the general population. Despite this, evidence regarding the incidence, elevated risk and predictive factors for violence-related deaths after release have not yet been synthesised. This information is important to inform the development of evidence-based approaches to effectively prevent deaths from violence in this population. This systematic review will synthesise the literature examining the crude mortality rates (CMRs), standardised mortality ratios (SMRs) and predictive factors for violence-related deaths among people released from incarceration.

Methods and analysis We searched key electronic health, social science and criminology databases (MEDLINE, PubMed, PsycINF0, Scopus, Web of Science, $\mathrm{CINCH}$, Criminal Justice Abstracts) for peer-reviewed cohort studies published in English on $14^{\text {th }}$ September 2020. Our primary outcome of interest is violence-related deaths occurring in the community following release from incarceration. We will not restrict study eligibility by year of publication or age of participants. The Methodological Standard for Epidemiological Research (MASTER) scale will be used to assess the quality of included studies. If there are sufficient studies and homogeneity between studies, we will conduct meta-analyses to calculate pooled estimates of CMRs, SMRs or predictive factors for violence-related deaths. If there is a sufficient number of included studies, meta-regression will be conducted to examine the influence of subgroups and methodological factors on the CMRs, SMRs or predictive factors. If the studies do not report sufficient data, or if there is substantial heterogeneity, findings will be presented in a narrative form.

Ethics and dissemination This review is exempt from ethics approval as it will synthesise findings from published studies that have already obtained ethics approval. Our findings will be disseminated through a peer-reviewed journal article, and national and international conference and seminar presentations. Trial registration details This study is registered with PROSPERO (CRD42020209422).

\section{INTRODUCTION}

Despite growing acknowledgement that incarceration is a costly and largely ineffective

\section{Strengths and limitations of this study}

- Findings from this systematic review will support the development of evidence-informed approaches to prevent violence-related deaths after release from incarceration.

- The systematic review will be reported in accordance with the Preferred Reporting Items for Systematic Reviews and Meta-Analysis (PRISMA), and is registered with PROSPER0 (CRD42020209422).

- The systematic review uses a comprehensive search strategy that was developed in consultation with a research institute librarian and comprises seven health, social science and criminology databases.

- To ensure the systematic review contains the highest standard of evidence possible for the outcomes, only published peer-reviewed cohort studies will be included.

- Only studies published in English will be included in the systematic review.

response to crime ${ }^{1-3}$ the number of people incarcerated globally has steadily increased at a rate that exceeds population growth and crime rates. ${ }^{4}$ Although the prison population in many countries increased rapidly prior to 2000, from 2000 to 2018 the global prison population grew by $24 \%$, with the largest increase in Oceania (87\%), the Americas $(41 \%)$ and Asia $(38 \%) .{ }^{5}$ It is currently estimated that on any given day there are approximately 11 million adults ${ }^{4}$ and 410000 children and adolescents (aged $<18$ years) imprisoned worldwide. ${ }^{6}$ The vast majority of these people will be released from custody, most after a relatively short period of incarceration. ${ }^{4}$

Young people (aged <25 years) and adults released from incarceration are more likely to die from preventable causes than the general population. Previous systematic reviews have shown that adults released from prison are at increased risk of all-cause ${ }^{78}$ and external-cause deaths, ${ }^{8}$ drug-related deaths ${ }^{9}$ and suicide. ${ }^{10}$ Additionally, the Mortality After Release from Incarceration Consortium 
(MARIC) has recently been established to examine mortality among people who have experienced incarceration internationally using individual participant data meta-analysis methods. ${ }^{11}$ However, so far only a protocol of the Consortium has been published. ${ }^{11}$

To our knowledge, no reviews have examined deaths among young people released from youth detention in detail. Two reviews that focussed on the health of young people in detention briefly considered suicide among young people after release, and found that these young people have an elevated risk of suicide in comparison with their community counterparts. ${ }^{12}{ }^{13}$ Cohort studies from high-income countries, such as the $\mathrm{USA}^{1415}$ and Australia, ${ }^{16}$ have found that young people released from youth detention are at increased risk of all-cause and external-cause deaths. Given the large and increasing number of adults and young people being incarcerated globally, there is a strong imperative to reduce excess mortality among these populations.

Incarceration disproportionately impacts people from disadvantaged communities, such as those who are socioeconomically deprived, from marginalised ethnic groups, or experiencing unemployment or homelessness. ${ }^{7-10}$ Incarceration may compound this existing disadvantage by further disrupting social support systems, employment, education and housing security. ${ }^{17} 18$ Experiencing these social and economic factors can lead to further social exclusion, poor health and an increased risk of death. ${ }^{19}$ Consistent with this, the prevalence of complex, co-occurring health conditions (eg, violence and abuse, mental health and substance use disorders, neurodevelopmental disabilities, chronic diseases and blood borne viruses) is higher among people who experience incarceration than in the general population. ${ }^{13}{ }^{20}$ As such, addressing the excess morbidity and mortality of people released from incarceration is an important health equity issue and will likely reduce health inequalities at the population level.

Young people ${ }^{14}$ and adults ${ }^{821}$ released from incarceration have a risk of dying from violence that far exceeds that in the general population. Zlodre and $\mathrm{Fazel}^{8}$ considered violence-related deaths in their 2012 systematic review of all-cause and external-cause mortality, and found that people released from incarceration were between 3 and 10 times more likely to die from violence compared with the general population. However, this study did not calculate pooled cause-specific estimates of crude mortality rates (CMRs) or standardised mortality ratios (SMRs) for violence-related death, possibly due to the limited number of included studies that examined this cause $(n=5 / 18)$. A preliminary search conducted while developing the search strategy for this review identified several new studies in this area ${ }^{1422-30}$ that have been published since Zlodre and Fazel's review was conducted almost a decade ago.

To our knowledge, no reviews have been conducted that have examined the predictors of violence-related death after release, or have examined violence-related death among young people released from incarceration. While
Zlodre and Fazel's ${ }^{8}$ review included some studies examining young people released from youth justice detention, ${ }^{1631}$ this setting was not an explicit part of the search strategy, and as such some potentially relevant studies may have been missed. A comprehensive review explicitly searching for violence-related deaths among young people released from youth justice detention is needed, given emerging evidence that these young people have a risk of dying from violence that far exceeds that of their counterparts in the general population. ${ }^{14}$

To our knowledge, the evidence on violence-related deaths among young people and adults released from incarceration has not been synthesised at a global level. As such, the global epidemiology of violence-related death after release from custody remains poorly understood. This knowledge is an important prerequisite to the development of evidence-informed approaches to prevent violence-related deaths in these populations. By conducting a systematic review, we aim to: (1) synthesise the peer-reviewed literature examining the CMRs, SMRs and predictive factors for violence-related deaths among people released from incarceration; and (2) calculate pooled estimates of these measures.

\section{METHODS AND ANALYSIS}

This protocol is reported in accordance with the Preferred Reporting Items for Systematic Reviews and Meta-Analysis for Protocols (PRISMA-P) ${ }^{32}$ and is registered with PROSPERO (CRD42020209422).

\section{Eligibility criteria \\ Participants}

We will include cohort studies examining people of any age who have been released from a youth justice detention facility or adult correctional institution (including both prisons and jails in the USA). Studies of people incarcerated outside of the criminal justice system (eg, in immigration detention) will be excluded.

\section{Outcome measure}

Our primary outcome will be violence-related death occurring in the community following release from incarceration. Consistent with the World Health Organization's (WHO) definition of interpersonal violence, we will define violence-related deaths as those resulting from the intentional use of physical force or power between two or more people that is not specifically intended to further any political, economic or social objectives of any group or cause. ${ }^{33}$ Consistent with international literature, ${ }^{31} 34$ deaths from legal intervention (ie, the killing of a person by a law-enforcement officer acting in the course of duty ${ }^{35}$ ) will be included in our definition of violencerelated deaths. We will not restrict study eligibility by the timeframe in which violence-related deaths are examined after release from incarceration (eg, within the first year after release). Studies examining deaths exclusively during a period of incarceration, or examining deaths 
both during and after incarceration (but which do not disaggregate by incarceration status at the time of death), will be excluded.

\section{Study design}

We will include published, peer-reviewed cohort studies (prospective and retrospective) of people released from incarceration. Previous reviews of this literature will not be included, as some of the included studies may not meet our eligibility criteria. However, the reference lists of these reviews will be searched to identify additional relevant studies which were not identified by the original search strategy. Study eligibility will not be restricted by year of publication. Only studies published in English will be included.

\section{Information sources and search strategy}

We searched key electronic health, social science and criminology databases (MEDLINE, PubMed, PsycINFO, Scopus, Web of Science, CINCH, Criminal Justice Abstracts) for peer-reviewed cohort studies published in English using variants and combinations of search terms relating to incarceration, death and violence on $14^{\text {th }}$ September 2020. The search strategy was developed in consultation with a librarian at the Murdoch Children's Research Institute in Melbourne, Australia. The MEDLINE search strategy is outlined in table 1. We used a version of Ovid's recommended observational study search filter ${ }^{36}$ adapted for each database to identify cohort studies. Reference lists of all included studies will be screened to identify any additional relevant studies.

\section{Study selection}

Studies identified through the database search will be imported into EndNote X8.2 $2^{37}$ and duplicates will be removed. The remaining citations will be uploaded into Covidence ${ }^{38}$ for screening. Titles and abstracts of potentially eligible studies will be reviewed by the lead researcher (MW), with $10 \%$ screened by a second researcher. Any uncertainty related to study inclusion will be resolved through discussion with a third research team member. The inter-rater reliability for the title and abstract screening will be calculated using Cohen's kappa statistic. ${ }^{39}$ Studies will be coded as either $0=$ Does not meet eligibility criteria, or $1=$ Meets eligibility criteria or the full-text article needs to be screened to confirm eligibility. After $10 \%$ of the citations have been screened, the eligibility criteria will be reassessed through discussion and consensus between all researchers to ensure that they are relevant to the studies that have been identified. If applicable, the updated eligibility criteria will be used for the remaining screening.

After title and abstract screening is completed, all remaining full-text articles will be independently screened by MW and a second researcher. Again, any conflicts related to study inclusion will be resolved through discussion with a third research team member. Where multiple
Table 1 MEDLINE search strategy

1. (offender* or detain* or imprison* or prison* or custod $^{\star}$ or incarcerat ${ }^{\star}$ or inmate ${ }^{\star}$ or criminal $^{\star}$ or (secure adj facility) or (secure adj facilities) or ((youth or juvenile) adj (detention* or delinquen*)) or (detention* adj (centre* or center* or unit or units or facilit*)) or jail* or gaol ${ }^{\star}$ or (penal adj (centre* or center ${ }^{\star}$ or unit or units or facilit $\left.{ }^{\star}\right)$ ) or (correction ${ }^{\star}$ adj (institut* or centre* or center ${ }^{\star}$ or unit or units or facilit*))).tw,kf

\begin{tabular}{|c|c|}
\hline 2. & Prisons/ \\
\hline 3. & Prisoners/ \\
\hline 4. & Juvenile Delinquency/ \\
\hline 5. & $\begin{array}{l}\text { (former or postrelease }{ }^{\star} \text { or post-release }{ }^{\star} \text { or release } \\
\text { or ex).tw,kf }\end{array}$ \\
\hline 6. & ( 1 or 2 or 3 or 4 ) and 5 \\
\hline 7. & 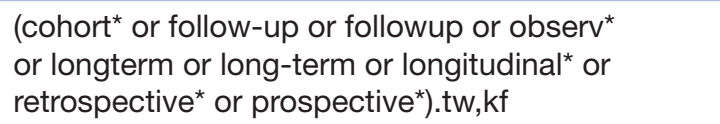 \\
\hline 8. & exp Cohort Studies/ or observational study/ \\
\hline 9. & 7 or 8 \\
\hline 10. & $\begin{array}{l}\text { (mortalit* or death }{ }^{\star} \text { or dead or fatal }{ }^{\star} \text { or lethal }{ }^{\star} \text { or } \\
\text { dying or } \text { die }^{\star} \text { or decease* or decedent }{ }^{\star} \text { ).tw,kf }\end{array}$ \\
\hline 11. & mortality/ \\
\hline 12. & "cause of death"/ \\
\hline 13. & fatal outcome/ \\
\hline 14. & Death/ \\
\hline 15. & mo.fs. \\
\hline 16. & 10 or 11 or 12 or 13 or 14 or 15 \\
\hline 17. & $\begin{array}{l}\text { (violen* or assault* or legal-intervention* or } \\
\text { murder* or manslaughter }{ }^{\star} \text { or homicide* or gun? or } \\
\text { firearm?).tw,kf }\end{array}$ \\
\hline 18. & exp Violence/ \\
\hline 19. & homicide/ \\
\hline 20. & firearms/ \\
\hline 21. & 17 or 18 or 19 or 20 \\
\hline 22. & 16 or 21 \\
\hline 23. & 6 and 9 and 22 \\
\hline
\end{tabular}

studies use the same data set, only the study with the longest follow-up period will be included.

\section{Data extraction}

Data extraction will be conducted by MW using a prespecified Excel form developed by the researchers. A second researcher will check the data extraction and amend any errors. The following information will be extracted from the included studies: author and year of publication, geographical location of the study, year/s of study, study design (eg, prospective, retrospective), sample selection techniques (eg, population vs selected samples such as people classified as 'serious violent offenders', people who inject drugs), cohort size, length of follow-up, whether the time in subsequent periods of 
incarceration during follow-up was removed from analysis (ie, interval truncation), attrition, number and proportion of violence-related deaths in the cohort, median/ mean age of people in the cohort overall and among those who died from violence, proportion of men and women in the cohort overall and among those who died from violence, proportion of people from a marginalised ethnic group in the cohort overall and among those who died from violence, definition of violence, record source (eg, death, court or police records), CMRs, SMRs and reference population, factors predictive of violencerelated death and the measure of association (eg, risk ratio, hazard ratio) and type of incarceration experienced (eg, youth justice detention, prison, jail).

\section{Risk of bias assessment}

The Methodological Standard for Epidemiological Research (MASTER) scale $^{40}$ will be used to assess the quality of included studies. The quality and risk of bias of each study will be assessed by MW, with any uncertainty being resolved through discussion and consensus with the research team. The MASTER scale has some advantages over other quality assessment scales as it has clearly defined domains of bias based in theory and empirical evidence that score studies' propensity towards bias, independent of the direction or magnitude of bias, relative to the highest scoring study in the review, as opposed to using quality scores as an absolute measure of bias. ${ }^{40}$ This scale ranks studies based on the number of safeguards against bias present in the study, with a higher number of safeguards indicating a lower probability of bias. ${ }^{40}$ Quality and risk of bias assessment will be conducted at the study level.

\section{Data synthesis}

We will provide a descriptive overview of the included studies, including the study year/s and geographical location, length of follow-up, type of incarceration and definition of violence.

If there is a sufficient number of studies which report the CMRs, SMRs or predictive factors for violence-related deaths after release from incarceration, we will conduct meta-analyses. If the CMRs are not reported in an included study, we will calculate them using the method outlined in Zlodre and Fazel, ${ }^{8}$ using the number of deaths and total person-years at risk or using median duration of follow-up to estimate total person-years. Heterogeneity between studies will be assessed using the $I^{2}$ statistic, with an $I^{2}$ value of $\geq 50 \%$ considered indicative of substantial heterogeneity. ${ }^{41}$

If there is a sufficient number of included studies, meta-regression will be conducted to examine the influence of subgroups and methodological factors on the CMRs, SMRs or predictive factors. ${ }^{42}$ The following factors may be considered: sex, marginalised ethnic group status (as defined by the included study; for example, Indigenous people/non-Indigenous people), type of incarceration facility, study design (eg, prospective, retrospective), length of follow-up, geographical location of the study, sample selection techniques (eg, population vs selected samples) and whether the time in subsequent periods of incarceration during follow-up was removed from analysis (ie, interval truncation). To examine the effect of study quality on the outcomes, sensitivity analyses will be conducted whereby we restrict analyses to studies rated as high-quality (defined as scoring above the median using the MASTER scale).

If meta-analyses are not possible due to a lack of sufficient data on the CMRs, SMRs or predictive factors in the included studies, or if there is substantial heterogeneity, a narrative synthesis will be conducted. The narrative synthesis will follow existing guidelines, ${ }^{43}$ which contain four main elements: developing a theoretical model (if relevant), preliminary synthesis of the included studies' findings, exploring relationships in the data and assessing the robustness of the synthesis.

\section{Patient and public involvement}

There was no patient and public involvement in the design of this study.

\section{ETHICS AND DISSEMINATION}

This systematic review is exempt from ethics approval as it will be carried out on published studies that have already obtained ethics approval. Our findings will be disseminated through a peer-reviewed journal article, and national and international conference and seminar presentations. We will also provide a plain language summary of our findings to relevant national and international bodies for dissemination.

\section{Author affiliations}

${ }^{1}$ Melbourne School of Population and Global Health, The University of Melbourne, Carlton, Victoria, Australia

${ }^{2}$ Centre for Adolescent Health, Murdoch Childrens Research Institute, Parkville, Victoria, Australia

${ }^{3}$ School of Population and Global Health, The University of Western Australia, Perth, Western Australia, Australia

${ }^{4}$ National Drug Research Institute, Curtin University, Perth, Western Australia, Australia

${ }^{5}$ Health Service and Population Research Department, Institute of Psychiatry, Psychology \& Neuroscience, King's College London, London, England, UK

${ }^{6}$ Melbourne School of Psychological Sciences, The University of Melbourne, Melbourne, Victoria, Australia

${ }^{7}$ Mater Research Institute-UQ, University of Queensland, Brisbane, Queensland, Australia

${ }^{8}$ Griffith Criminology Institute, Griffith University, Brisbane, Queensland, Australia ${ }^{9}$ School of Public Health and Preventive Medicine, Monash University, Melbourne, Victoria, Australia

Correction notice This article has been corrected since it first published. The provenance and peer review statement has been included.

Twitter Melissa Willoughby @Melwillo27, Jesse T Young @jtyoung_edu and Stuart A Kinner @KinnerStuart

Contributors MW developed the original research proposal. MW, JY, MS, RB and SK contributed to the design of the project. MW developed the search strategy with input from JY, MS, RB and SK. MW wrote the initial draft manuscript. MW, JY, MS, $\mathrm{RB}, \mathrm{EJ}$ and SK contributed significantly to drafting and editing the manuscript. All authors approved the final manuscript. 
Funding MW is supported by a National Health and Medical Research Council (NHMRC) Postgraduate Scholarship (GNT1151103). JY receives salary and research support from a NHMRC Investigator Grant (GNT1178027). MS is a recipient of an Australian Research Council Future Fellowship (project number FT180100075) funded by the Australian Government. SK receives salary support from a NHMRC Senior Research Fellowship (GNT1078168). Apart from the funding bodies listed above, this research received no specific grant from any funding agency in the public, commercial or not-for-profit sectors.

\section{Competing interests None declared.}

Patient and public involvement Patients and/or the public were not involved in the design, or conduct, or reporting, or dissemination plans of this research.

Patient consent for publication Not required.

Provenance and peer review Not commissioned; externally peer reviewed.

Open access This is an open access article distributed in accordance with the Creative Commons Attribution Non Commercial (CC BY-NC 4.0) license, which permits others to distribute, remix, adapt, build upon this work non-commercially, and license their derivative works on different terms, provided the original work is properly cited, appropriate credit is given, any changes made indicated, and the use is non-commercial. See: http://creativecommons.org/licenses/by-nc/4.0/.

\section{ORCID iDs}

Melissa Willoughby http://orcid.org/0000-0002-4360-2605

Jesse T Young http://orcid.org/0000-0001-5702-372X

\section{REFERENCES}

1 Queensland Productivity Commission. Inquiry into imprisonment and recidivism. Queensland, Australia Queensland Productivity Commission; 2019. https://www.qpc.qld.gov.au/inquiries/ imprisonment/

2 Mears DP, Cochran JC, Bales WD. Gender differences in the effects of prison on recidivism. J Crim Justice 2012;40:370-8.

3 Cullen FT, Jonson CL, Nagin DS. Prisons do not reduce recidivism: the high cost of ignoring science. The Prison Journal 2011:91:48S-65.

4 Jacobson J, Heard C, Fair H. Prison: evidence of its use and overuse from around the world. London Institute for Criminal Policy Research; 2017. https://www.prisonstudies.org/sites/default/files/ resources/downloads/global_imprisonment_web2c.pdf

5 World Prison Brief. World prison population list. London Institute for Crime and Justice Policy Research; 2018. https://www.prisonstudies. org/sites/default/files/resources/downloads/wppl_12.pdf

6 United Nations. Global study on children deprived of liberty. Geneva UN; 2019. https://childrendeprivedofliberty.info/wp-content/uploads/ 2019/08/GA-report_GSDCL.pdf

7 Kinner SA, Forsyth S, Williams G. Systematic review of record linkage studies of mortality in ex-prisoners: why (good) methods matter. Addiction 2013;108:38-49.

8 Zlodre J, Fazel S. All-Cause and external mortality in released prisoners: systematic review and meta-analysis. Am J Public Health 2012;102:e67-75.

9 Merrall ELC, Kariminia A, Binswanger IA, et al. Meta-Analysis of drug-related deaths soon after release from prison. Addiction 2010;105:1545-54.

10 Jones D, Maynard A. Suicide in recently released prisoners: a systematic review. Mental Health Practice 2013;17:20-7.

11 Borschmann R, Tibble H, Spittal MJ, et al. The mortality after release from incarceration Consortium (MARIC): protocol for a multi-national, individual participant data meta-analysis. Int J Popul Data Sci 2020;5.

12 Golzari M, Hunt SJ, Anoshiravani A. The health status of youth in juvenile detention facilities. J Adolesc Health 2006;38:776-82.

13 Borschmann R, Janca E, Carter A, et al. The health of adolescents in detention: a global scoping review. Lancet Public Health 2020;5:e114-26.

14 Teplin LA, Jakubowski JA, Abram KM, et al. Firearm homicide and other causes of death in delinquents: a 16-year prospective study. Pediatrics 2014;134:63-73.

15 Aalsma MC, Lau KSL, Perkins AJ, et al. Mortality of youth offenders along a continuum of justice system involvement. Am J Prev Med 2016;50:303-10.
16 Coffey C, Veit F, Wolfe R, et al. Mortality in young offenders: retrospective cohort study. BMJ 2003;326:1064.

17 Aldridge RW, Story A, Hwang SW, et al. Morbidity and mortality in homeless individuals, prisoners, sex workers, and individuals with substance use disorders in high-income countries: a systematic review and meta-analysis. Lancet 2018;391:241-50.

18 Hughes N, Ungar M, Fagan A, et al. Health determinants of adolescent criminalisation. Lancet Child Adolesc Health 2020;4:151-62.

19 Marmot M. Inclusion health: addressing the causes of the causes. Lancet 2018;391:186-8.

20 Kinner SA, Young JT. Understanding and improving the health of people who experience incarceration: an overview and synthesis. Epidemiol Rev 2018;40:4-11.

21 Binswanger IA, Stern MF, Deyo RA. Release from prison a high risk of death. New Eng J Med 2007;356:157-65.

22 Willoughby M, Spittal MJ, Borschmann R, et al. Violence-related deaths among people released from prison: a data linkage study. $J$ Interpers Violence 2020;0886260520905546:088626052090554.

23 Lize SE, Scheyett AM, Morgan CR, et al. Violent death rates and risk for released prisoners in North Carolina. Violence Vict 2015;30:1019-36.

24 Lim S, Seligson AL, Parvez FM, et al. Risks of drug-related death, suicide, and homicide during the immediate post-release period among people released from New York City jails, 2001-2005. Am J Epidemiol 2012;175:519-26.

25 Binswanger IA, Blatchford PJ, Mueller SR, et al. Mortality after prison release: opioid overdose and other causes of death, risk factors, and time trends from 1999 to 2009. Ann Intern Med 2013;159:592-600.

26 Chang Z, Lichtenstein P, Larsson H. Substance use, psychiatric disorders, and mortality after release. The Lancet Psy 2015;2:422-30.

27 Degenhardt L, Larney S, Kimber J, et al. The impact of opioid substitution therapy on mortality post-release from prison: retrospective data linkage study. Addiction 2014;109:1306-17.

28 Huber F, Merceron A, Madec Y, et al. High mortality among male HIV-infected patients after prison release: ART is not enough after incarceration with HIV. PLoS One 2017;12:e0175740.

29 Spaulding AC, Sharma A, Messina LC, et al. A comparison of liver disease mortality with HIV and overdose mortality among Georgia prisoners and releasees: a 2-decade cohort study of prisoners incarcerated in 1991. Am J Public Health 2015;105:e51-7.

30 Brinkley-Rubinstein L, Sivaraman J, Rosen DL, et al. Association of restrictive housing during incarceration with mortality after release. JAMA Netw Open 2019;2:e1912516-e.

31 Teplin LA, McClelland GM, Abram KM, et al. Early violent death among delinquent youth: a prospective longitudinal study. Pediatrics 2005;115:1586-93.

32 Moher D, Shamseer L, Clarke M, et al. Preferred reporting items for systematic review and meta-analysis protocols (PRISMA-P) 2015 statement. Syst Rev 2015;4:1.

33 Rutherford A, Zwi AB, Grove NJ, et al. Violence: a glossary. J Epidemiol Community Health 2007;61:676-80.

34 Ezell ME, Tanner-Smith EE. Examining the role of lifestyle and criminal history variables on the risk of homicide victimization. Homicide Stud 2009;13:144-73.

35 World Health Organization. Classification of diseases (ICD), 2018 Available: http://www.who.int/classifications/icd/en/

36 Wolters Kluwer. Ovid Expert Searches - Health Science, 2019. Available: http://resourcecenter.ovid.com/site/resources/expert_ search/healthexp.html\#OvidFilters

37 Clarivate Analytics. Endnote X8, 2018. Available: https://endnote. com/ [Accessed 8 Sep 2020]

38 Covidence. Available: https://www.covidence.org/ [Accessed 8 Sep 2020].

39 McHugh ML. Interrater reliability: the kappa statistic. Biochem Med 2012;22:276-82.

40 Stone JC, Glass K, Clark J, et al. A unified framework for bias assessment in clinical research. Int $J$ Evid Based Healthc 2019;17:106-20.

41 Higgins JPT, Thompson SG. Quantifying heterogeneity in a metaanalysis. Stat Med 2002;21:1539-58.

42 Cochrane Training. Cochrane Handbook for systematic reviews of interventions, 2019. Available: https://training.cochrane.org/ handbook/current

43 Popay J, Roberts H, Sowden A. Guidance on the conduct of narrative synthesis in systematic reviews: a product from the ESRC methods program, 2006. Available: https://citeseerx.ist.psu.edu/ viewdoc/download?doi=10.1.1.178.3100\&rep=rep1\&type=pdf 